\title{
Multiple drug resistance bacterial isolates and associated factors among urinary stone patients at the University of Gondar Comprehensive Specialized Hospital, Northwest Ethiopia
}

Desie Kasew ${ }^{1 *}$, Setegn Eshetie ${ }^{1}$, Abeje Diress ${ }^{2}$, Zerubabiel Tegegne ${ }^{2}$ and Feleke Moges ${ }^{1}$

\begin{abstract}
Background: The urinary stone and urinary tract infection (UTI) are invariably associated and are frequent causes of morbidity. Date on burden of UTI among urinary stone patients is lacking in Ethiopia. This study was aimed to assess bacterial profile, antimicrobial susceptibility and associated factors among urinary stone patients at the University of Gondar Comprehensive Specialized Hospital.

Methods: An institution based cross sectional study was conducted. Basic sociodemographic data were collected using a structured questionnaire. Bacterial identification of uropathogens and drug susceptibility testing were done following standard microbiological techniques. The data were entered and analyzed using SPSS version-23. Bivariate and multivariate logistic regressions were used to identify possible associated risk factors. Results with $P$ value $<0.05$ was considered statistically significant.

Result: A total of 300 urinary stone patients were enrolled. Of these, 153 (51\%) were male and 261(87\%) were urban residents. The overall prevalence of urinary tract infection was 49 (16.3\%) (95\% Cl 12-21\%). A high level of resistance was observed to ampicillin, penicillin and trimethoprim-sulfamethoxazole while majority of isolates were most sensitive to nitrofurantoin and ciprofloxacin. Multi-drug resistant isolates were 16/49 (32.7\%), 75\% of them being Enterobacteriaceae isolates. More than one-third 9/26 (34.6\%) of Gram-negative isolates were Extended Spectrum Beta-Lactamase (ESBL) producing E. coli and K. pneumoniae. Being female, history of urinary tract infection and history of drug use were the independent risk factors.

Conclusion: Most of the bacterial isolates from urinary stone patients were resistant to ampicillin, penicillin and trimethoprim-sulfamethoxazole. E. coli and K. pneumoniae were the most common extended spectrum beta-lactamase producing isolates. Sex, history of urinary tract infection and previous drug use were found to be risk factors. Routine diagnosis of urinary stone patients for urinary tract infection should be promoted and further researches are encouraged.
\end{abstract}

\footnotetext{
*Correspondence: dessalegnkassaw83@gmail.com; Desie.Kasaw@uog.edu.et

${ }^{1}$ Department of Medical Microbiology, School of Biomedical

and Laboratory Sciences, College of Medicine and Health Sciences,

University of Gondar, P.O. Box 196, Gondar, Ethiopia

Full list of author information is available at the end of the article
}

(c) The Author(s) 2021. Open Access This article is licensed under a Creative Commons Attribution 4.0 International License, which permits use, sharing, adaptation, distribution and reproduction in any medium or format, as long as you give appropriate credit to the original author(s) and the source, provide a link to the Creative Commons licence, and indicate if changes were made. The images or other third party material in this article are included in the article's Creative Commons licence, unless indicated otherwise in a credit line to the material. If material is not included in the article's Creative Commons licence and your intended use is not permitted by statutory regulation or exceeds the permitted use, you will need to obtain permission directly from the copyright holder. To view a copy of this licence, visit http://creativecommons.org/licenses/by/4.0/ The Creative Commons Public Domain Dedication waiver (http://creativeco mmons.org/publicdomain/zero/1.0/) applies to the data made available in this article, unless otherwise stated in a credit line to the data. 
Keywords: Urinary stone, Multiple drug resistance, Urinary tract infection

\section{Background}

Urinary stone and urinary tract infections (UTI) are constantly associated complaints of the urinary system [1]. Urinary stones also known as urolithiasis [2] are hard masses produced in the urinary tract and cause infection, pain, bleeding or obstruction. Multiple types of stones such as oxalate, uric acid, cysteine, or struvite stone can be produced in the urinary tract [3]. Infection stones include magnesium ammonium phosphate or struvite (which accounts to $10-15 \%$ of urinary stone), carbonate apatite and ammonium urate. Urease producing bacteria such as Proteus are responsible for production of urease enzyme which alkalinizes urine and produce these stones $[4,5]$. Moreover, ammonia and oxalate stones damage the urothelial layer facilitating microbial invasion [6].

E. coli, a urease negative bacterium is the most common cause of UTI and dominant isolate in urinary stones and urine cultures. Its existence in stone indicates either urease negative bacteria have a role in stone formation or urease producing bacteria transiently infect and lost after stone formation [7]. Urease producing bacteria include Proteus, Morganella, Pseudomonas, Providencia, Klebsiella and S. aureus and are common causes of UTI [8]. P. mirabilis is the prominent urease producing species and cause of struvite calculi [9]. Bacteria incrusted in or attached on the surface of stone forming biofilm and cause recurrent and multidrug resistant (MDR) UTI [10]. The rate of UTI among urolithiasis patients ranges from 7 to $60 \%$ [11] with the coincidence of these comorbidities results in complications such as renal failure and death [12].

Urinary stone disease is one of the worldwide threats which has been increasing particularly in the last 15 years $[13,14]$. Factors such as sex, previous history of UTI, condom use, vaginal infection, extremes of age, anatomical abnormalities like congenital urinary tract malformations, urinary obstruction, catheterization, resent sexual activity, comorbidities like urinary stone, diabetes and obesity $[8,15,16]$, hospitalization, antibiotic use are risks factors to UTI [17]. Information about the burden of UTI among patients with urinary calculi is lacking in Ethiopia. This study is aimed to investigate bacterial uropathogens, antimicrobial resistance and associated factors among Urinary stone patients at the University of Gondar Comprehensive Specialized Hospital, Gondar Ethiopia.

\section{Materials and methods Study area, design, and period}

A prospective cross-sectional study was conducted from January to April, 2019, at the University of Gondar Comprehensive Specialized Hospital. The hospital is located in Gondar town, Amhara regional state, Northwest Ethiopia, situated at $742 \mathrm{~km}$ from the capital city of Ethiopia, Addis Ababa. The hospital is a tertiary level teaching and referral hospital catering more than 500 beds for inpatients and rendering referral health services for over 5 million inhabitants in Northwest Ethiopia.

Population All patients who have visited the hospital during the study period and clinically suspected of urinary stone disease were the source population while patients with ultrasound confirmed urinary stone were study population. All the study participants were enrolled from the outpatient department.

Exclusion criteria Patients with history of antibiotic use in the last 2 weeks prior to diagnosis and during the data collection period were excluded or denied from the study because those who have been taking antibiotics may have culture negative due to their resent drug use. All patients who had urinary system complains, were confirmed to have urinary stone with ultrasound and not on antibiotics treatment in the last 2 weeks as well as during the study period and willing to be included were eligible to this study.

\section{Sample size and sampling methods}

The sample size was calculated by a single population proportion formula taking $50 \%$ prevalence since previous study involving urinary stone patients was not available.

$$
\begin{aligned}
& \mathrm{n}=\frac{\mathrm{z}_{\alpha / 2}^{2} \mathrm{p}(1-\mathrm{p})}{\mathrm{d}^{2}} \\
& =\frac{1.96^{2} * 0.5(1-0.5)}{(0.05)^{2}}=384
\end{aligned}
$$

Using reduction formula as the total population based on the same period of the last year's record, estimated population was 1350 , the final sample size was

$$
\begin{aligned}
& \mathrm{n}_{\mathrm{f}}=\frac{\mathrm{n} * \mathrm{~N}}{\mathrm{n}+\mathrm{N}} \\
& \mathrm{n}_{\mathrm{f}}=\frac{384 * 1350}{384+1350}=299
\end{aligned}
$$


where $\mathrm{N}$ is the total population, $\mathrm{n}_{\mathrm{f}}$-the final sample size, $\mathrm{Z}_{\alpha / 2}$-the standard normal deviation, at $95 \%$ confidence level $=1.96, \mathrm{p}-$ The prevalence $=0.5 ; 1-\mathrm{p}=0.5$ and $\mathrm{d}-$ The desired degree of accuracy $=0.05$.

\section{Data collection and laboratory methods}

A total of 300 study participants with urinary stone were enrolled in the study using convenient sampling technique. Sociodemographic and clinical features of study participants such as age, gender, residence, history of UTI and drug use, stone size and its position, presence of stone at multiple sites, condom use, catheterization and presence of comorbidites like history of diabetes, HIV were colleceted using structured questionaire. Clean catch mid-stream urine was collected in a sterile wide mouthed urine cup with prior adiquate instruction of the participant (Additional file 1).

\section{Culture and microbiological identification}

Early morning mid-stream urine sample was collected and transported to medical bacteriology laboratory; inoculated in Cysteine lactose electrolyte deficient (CLED) agar (BIOMARK ${ }^{\mathrm{R}}$ Laboratories, India) within two hours and incubated for $18-24 \mathrm{~h}$ at $35-37^{\circ} \mathrm{C}$. A $0.001 \mathrm{~mL}$ inoculating loop was used to inoculate the urine specimen. Colonies with significant bacteriuria $\left(\geq 10^{5} \mathrm{CFU} / \mathrm{mL}\right)$ were subcultured on blood agar plate (BAP) and MacConkey agar for isolation of a single species and further characterization by hemolysis on BAP, lactose fermentation on MacConkey agar. Gram staining and biochemical tests such TSI, motility test, indole test, citrate test, urease test, Lysine decarboxylase, catalase, coagulase tests, novobiocin sensitivity, and Bile Esculin (BIOMARK $^{\mathrm{R}}$ Laboratories, India) hydrolysis test were used for species identification [18].

\section{Antimicrobial susceptibility testing}

Antimicrobial susceptibility testing (AST) was performed using Kirby-Bauer disc diffusion method on Muller-Hinton agar (BIOMARK ${ }^{\mathrm{R}}$ Laboratories, India). Selected antimicrobial agents such as Ampicillin $(10 \mu \mathrm{g})$, Amoxicillin-clavulanate $(20 / 10 \mu \mathrm{g})$, Ciprofloxacin $(5 \mu \mathrm{g})$, Tobramycin $(10 \mu \mathrm{g})$, Gentamicin $(10 \mu \mathrm{g})$, Cefixime $(5 \mu \mathrm{g})$, Cefoxitin $(30 \mu \mathrm{g})$, Cefuroxime $(30 \mu \mathrm{g})$, Nitrofurantoin $(300 \mu \mathrm{g})$, Rifampin $(5 \mu \mathrm{g})$, Tetracycline $(30 \mu \mathrm{g})$, Penicillin 10 units, Norfloxacin $(10 \mu \mathrm{g})$, Vancomycin $(30 \mu \mathrm{g})$, Trimethoprim-sulphamethoxzole (1.25/23.75 $\mu \mathrm{g})$ (Abtek Biologicals Ltd, United Kingdom) and Cefotaxime $(30 \mu \mathrm{g})$, Ceftazidime $(30 \mu \mathrm{g})$, Cefotaxime-Clavulanate $(30 / 10 \mu \mathrm{g})$, Ceftazidime-Clavulanate $(30 / 10 \mu \mathrm{g})(\mathrm{HiMe}-$ dia Laboratories Pvt Ltd India) were used for antimicrobial susceptibility test [19]. Zones of inhibition were measured after $16-18 \mathrm{~h}$ of incubation at $37^{\circ} \mathrm{C}$ and classified as susceptible or resistant. Isolates intermediate between susceptible and resistant were considered as resistant [20]. Isolates resistant to three or more classes of antibiotics were considered as MDR [21].

\section{Extended spectrum beta lactamase detection}

First screening for ESBL production was done for Cefotaxime and Ceftazidime $30 \mu \mathrm{g}$ each. Isolates with zone of inhibition $\leq 27 \mathrm{~mm}$ for cefotaxime and $\leq 22 \mathrm{~mm}$ for ceftazidime were phenotypically confirmed for ESBL production by using Ceftazidime-clavulanic acid $(30 \mu \mathrm{g} / 10 \mu \mathrm{g})$, Cefotaxime-clavulanic acid $(30 \mu \mathrm{g} / 10 \mu \mathrm{g})$ with their respective single disks using combined disk test. The suspension of screened isolates was prepared and inoculated on MHA. The target antibiotics were then placed at least $25 \mathrm{~mm}$ apart from each other followed by 16-18 $\mathrm{h}$ of incubation at $35{ }^{\circ} \mathrm{C} \pm 2{ }^{\circ} \mathrm{C}$ and zone of inhibition was measured. At least $5 \mathrm{~mm}$ difference to either of the combined drugs and their respective single disks was considered as ESBL producing species [19].

Quality control The questionnaire was pretested for standardization and clearity. The culture media were checked for sterility and performance prior to inoculation by incubating $5 \%$ of the newly prepared media overnight. The reagents for Gram's stain were also assured for their expiry date and staining. The control strains $E$. coli ATCC25922 and S. aureus ATCC25923 were used to control performance of media and antibiotic disks. Furthermore, K. pneumoniae ATCC 700603 and E. coli ATCC25922 were used as a positive and negative control for ESBL production respectively. The suspension was standardized against 0.5 McFarland standards [19].

Data processing and analysis Data were entered and analyzed using SPSS version 23 software. Discriptive statistics such as frequency and percentage were determined. The binary mogistic regression model was used. The assumption Hosmer-Lemeshow goodness of fit as well as cox and snell and Nagelkerke R squire valus were checked and the model was fit. Bivariate and multivariate logistic regression analysis were carried out with enter method to determine statistically significant association and odds ratio at $95 \%$ confidence interval was computed. Variables with a $P$ value $<0.2$ in bivariate logistic regression analysis were entered to multivariate logestic regression analysis $[22,23]$. Variables with $P$ value $<0.05$ in multivariate logistic regression analysis were considered as statistically significant. 
Table 1 Sociodemographic characteristics of urinary stone patients at the University of Gondar Comprehensive Specialized Hospital, January to April 2019

\begin{tabular}{|c|c|}
\hline Variables & Frequency (\%) \\
\hline \multicolumn{2}{|l|}{ Sex } \\
\hline Male & $153(51)$ \\
\hline Female & $147(49)$ \\
\hline \multicolumn{2}{|l|}{ Age } \\
\hline$<20$ years & $23(7.7)$ \\
\hline $20-29$ & $123(41)$ \\
\hline $30-39$ & $97(32.3)$ \\
\hline $40-49$ & $32(10.7)$ \\
\hline$\geq 50$ & $25(8.3)$ \\
\hline \multicolumn{2}{|l|}{ Residence } \\
\hline Urban & $261(87)$ \\
\hline Rural & $39(13)$ \\
\hline \multicolumn{2}{|l|}{ Educational level } \\
\hline Cannot read and write & $35(11.7)$ \\
\hline Elementary & $71(23.7)$ \\
\hline High school and preparatory & $60(20)$ \\
\hline College and above & $134(44.7)$ \\
\hline \multicolumn{2}{|l|}{ Family monthly income (ETB) } \\
\hline$<1000$ & $54(18)$ \\
\hline $1001-2000$ & $70(23.3)$ \\
\hline$>2000$ & $176(58.7)$ \\
\hline \multicolumn{2}{|l|}{ Sexual activity } \\
\hline Within 2 days & $43(14.3)$ \\
\hline Within 7 days & $84(28)$ \\
\hline \multicolumn{2}{|l|}{ Pregnancy (for females) } \\
\hline Yes & $6(2)$ \\
\hline No & $141(47)$ \\
\hline \multicolumn{2}{|l|}{ Use of birth control mechanisms } \\
\hline Condom & $6(2)$ \\
\hline No & $141(46.3)$ \\
\hline \multicolumn{2}{|l|}{ Body mass index } \\
\hline$<18.5$ & $40(13.3)$ \\
\hline $18.5-24.5$ & $211(70.3)$ \\
\hline$>24.5$ & $49(16.3)$ \\
\hline
\end{tabular}

\section{Result}

Sociodemographic and clinical characteristics of patients with urinary stone

Of the 300 urinary stone patients enrolled in the present study, 153(51\%) were male, 123(41\%) were in age group 20-29 years, 261(87\%) were urban dwellers and 134(44.7\%) were patients with educational level of college and above. More than half, 176(58.7\%) of participants had family monthly income 2000 Ethiopian birr and above (Table 1$)$. Thirty-three (11\%) of study participants had history of UTI, 25(8.3\%) of them had stone size
Table 2 Clinical characteristics of urinary stone patients at the University of Gondar Comprehensive Specialized Hospital, January to April 2019

\begin{tabular}{|c|c|}
\hline Variable & Frequency (\%) \\
\hline \multicolumn{2}{|l|}{ History of UTI } \\
\hline Yes & $33(11)$ \\
\hline No & 267(89) \\
\hline \multicolumn{2}{|l|}{ Urinary blockage } \\
\hline Yes & 9(3) \\
\hline No & $291(97)$ \\
\hline \multicolumn{2}{|l|}{ History of catheterization } \\
\hline Yes & $4(1.3)$ \\
\hline No & 296(98.7) \\
\hline \multicolumn{2}{|l|}{ Stone size } \\
\hline$<5 \mathrm{~mm}$ & 275(91.7) \\
\hline$\geq 5 \mathrm{~mm}$ & $25(8.3)$ \\
\hline \multicolumn{2}{|l|}{ Stones at multiple locations } \\
\hline Yes & $75(25)$ \\
\hline No & $225(75)$ \\
\hline \multicolumn{2}{|l|}{ Location of stone } \\
\hline Renal & 282(94) \\
\hline Renal and/or extra renal & $18(6)$ \\
\hline \multicolumn{2}{|l|}{ History of chronic disease } \\
\hline Yes & $21(7)$ \\
\hline No & 279(93) \\
\hline \multicolumn{2}{|l|}{ History of drug use } \\
\hline Yes & $172(57.3)$ \\
\hline No & $128(42.7)$ \\
\hline \multicolumn{2}{|l|}{ Drug type used } \\
\hline Antibiotics & $166(55.3)$ \\
\hline Antibiotics, steroids and HAART & $7(2.3)$ \\
\hline \multicolumn{2}{|l|}{ Antibiotic use } \\
\hline Within the past one month & $70(23.3)$ \\
\hline Within the past 3 months & $41(13.7)$ \\
\hline \multicolumn{2}{|l|}{ History of hospitalization } \\
\hline Yes & $17(5.7)$ \\
\hline No & 283(94.3) \\
\hline
\end{tabular}

Extra renal-(Kidney and ureter, Kidney and bladder, or Kidney and urethra)

of $5 \mathrm{~mm}$ and above. Nine (3\%) of the participants had history of urinary blockage and 166(55.3\%) of them had history of antibiotic use while 17(5.7\%) were hospitalized previously (Table 2).

The prevalence of bacterial uropathogens among patients with urinary stone

Forty-nine $49 / 300(16.3 \%)$ (95\% CI 12-21\%) of the urinary stone patients had UTI. The most common cause of UTI was E. coli $14 / 49(28.6 \%)$ followed by S. saprophyticus and Enterococcus species 8/49(16.3\%) each, K. pneumoniae and S. aureus 7/49(14.3\%) each (Fig. 1). 


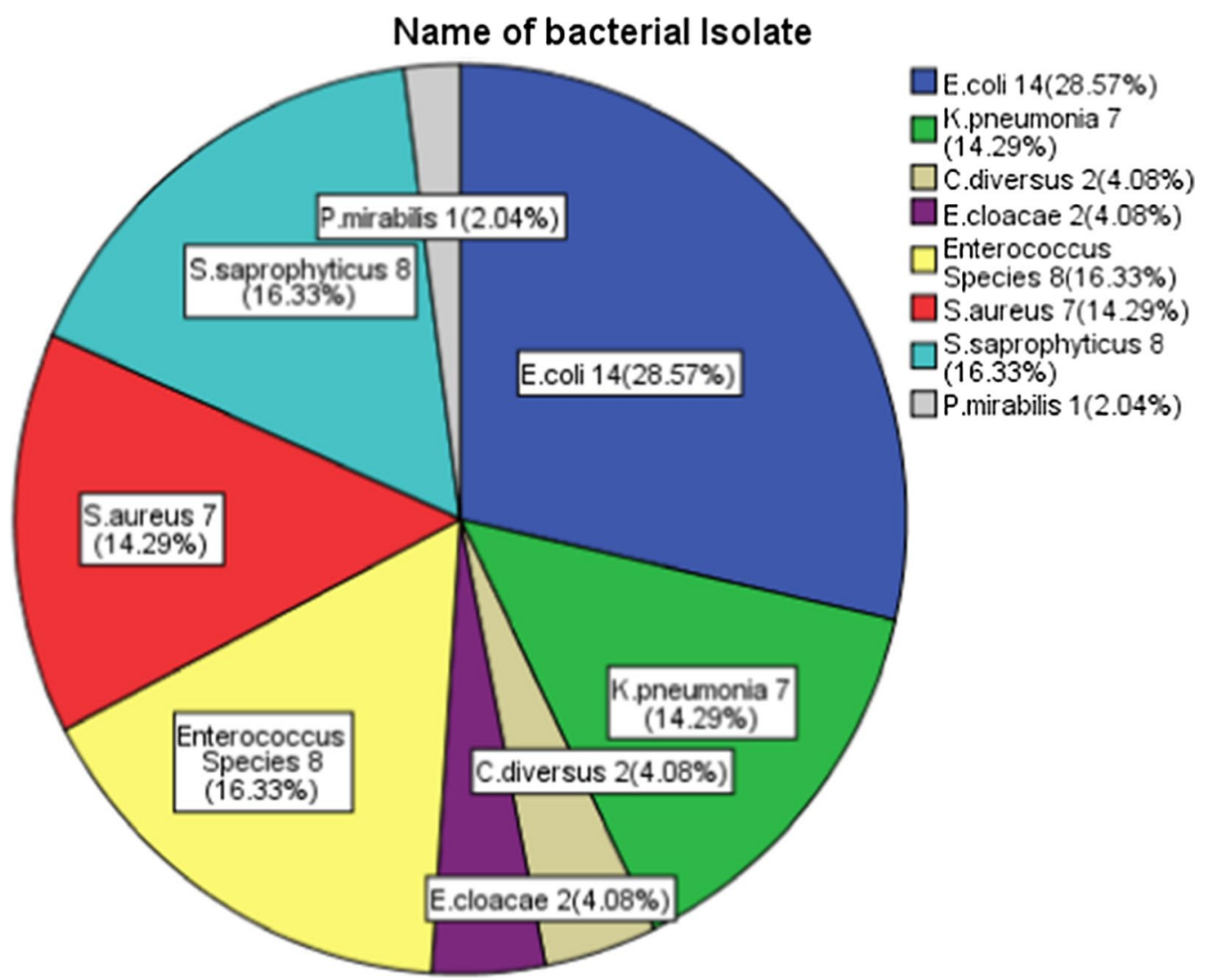

Fig. 1 The proportion of bacterial uropathogens isolated from Urinary stone patients at the University of Gondar Comprehensive Specialized Hospital, January to April 2019

\section{Antimicrobial susceptibility of bacterial uropathogens among patients with urinary stone}

Majority of Gram-negative isolates were resistant to ampicillin 25/26 (96.2\%), amoxicillin-clavulanate 18/26 (69.2\%) while $46 / 49$ (94\%) and 44/49(90\%) of the Gramnegative isolates were susceptible to nitrofurantoin and ciprofloxacin respectively. All S. saprophyticus 8(100\%) and $6 / 7(85.7 \%)$ of $S$. aureus were susceptible to gentamycin. Two 2/8(25\%) S. saprophyticus and 1/7 (14.3\%) of $S$. aureus isolates were resistant to cefoxitin (surrogate marker for methicillin resistance), and one vancomycin resistant Enterococcus was isolated (Table 3).

\section{MDR and ESBL producing uropathogenic isolates among patients with urinary stone}

Of the total uropathogens, 16/49(32.7\%) were MDR and $75 \%$ of which were Enterobacteriaceae. The most common MDR species were E. coli 7/49(14.3\%), followed by K. pneumoniae 3/49(6.12\%). Furthermore, from a total of 26 Enterobacteriaceae 9/26(34.6\%) isolates were confirmed ESBL producing species. These confirmed ESBL producing isolates were E. coli 6/26 (23.1\%) and K. pneumoniae 3/26(11.5\%) (Table 4).

\section{Factors associated with urinary tract infection among patients with urinary stone}

Among the variables, sex, age, education, monthly income, history of UTI, blockage of urinary system, stone size, stone location in the urinary system, history of chronic disease and drug use, sexual activity, history of hospitalization, and body mass index had $P$ value $<0.2$ in bivariate analysis and were computed by multivariate logistic regression analysis to check statistically significant association by controlling possible confounders. The result of multivariate logistic regression showed that being female, history of UTI, drug use had a $P$ value of $<0.05$; which indicates statistically significant association with patients having urinary stone diseases (Table 5).

\section{Discussion}

The overall prevalence of UTI among patients with urinary stone was $49 / 300(16.3 \%)$ (95\% CI 12-21\%). This result is similar to reports by Gutierrez et al. $16.2 \%$ 
Table 3 Antimicrobial Susceptibility Pattern of Bacterial Isolates from Urinary stone Patients at the UOGCSH, January to April 2019

\begin{tabular}{|c|c|c|c|c|c|c|c|c|}
\hline \multirow{2}{*}{$\begin{array}{l}\text { Antibiotic } \\
\text { used }\end{array}$} & \multicolumn{8}{|c|}{ Species of isolate with their antibiotic susceptibility } \\
\hline & E. coli N (\%) & $\begin{array}{l}\text { K. } \\
\text { pneumoniae } \\
\mathrm{N}(\%)\end{array}$ & $\begin{array}{l}\text { C. diversus N } \\
\text { (\%) }\end{array}$ & E. cloacae $\mathrm{N}(\%)$ & $\begin{array}{l}\text { P. mirabilis N } \\
\text { (\%) }\end{array}$ & $\begin{array}{l}\text { Enterococci N } \\
(\%)\end{array}$ & $\begin{array}{l}\text { S. } \\
\text { saprophyticus } \\
\mathrm{N}(\%)\end{array}$ & S. aureus $\mathrm{N}(\%)$ \\
\hline \multicolumn{9}{|l|}{ AMP } \\
\hline$S$ & & $1(14.3)$ & & & & $2(25)$ & & \\
\hline $\mathrm{R}$ & 14(100) & $6(85.7)$ & $2(100)$ & $2(100 \%)$ & $1(100)$ & $6(75)$ & & \\
\hline \multicolumn{9}{|l|}{ AMC } \\
\hline S & $2(14.3)$ & $4(57.1)$ & & $1(50)$ & $1(100)$ & & & \\
\hline $\mathrm{R}$ & $12(85.7)$ & $3(42.9)$ & $2(100)$ & $1(50)$ & & & & \\
\hline \multicolumn{9}{|l|}{ CTX } \\
\hline S & $7(50)$ & $6(85.7)$ & $1(50)$ & $2(100)$ & $1(100)$ & & & \\
\hline $\mathrm{R}$ & 7(50) & $1(14.3)$ & $1(50)$ & & & & & \\
\hline \multicolumn{9}{|l|}{ CAZ } \\
\hline S & $14(100)$ & $6(85.7)$ & $1(50)$ & $2(100 \%)$ & $1(100)$ & & & \\
\hline $\mathrm{R}$ & & $1(14.3)$ & $1(50)$ & & & & & \\
\hline \multicolumn{9}{|l|}{ FOX } \\
\hline S & 13(92.9) & $6(85.7)$ & $2(100)$ & $2(100 \%)$ & $1(100)$ & & $5(62.5)$ & $6(85.7)$ \\
\hline R & $1(7.1)$ & $1(14.3)$ & & & & & $3(37.3)$ & $1(14.3)$ \\
\hline \multicolumn{9}{|l|}{ CFM } \\
\hline S & $12(85.7)$ & $4(57.1)$ & $1(50)$ & $2(100)$ & $1(100)$ & & & \\
\hline $\mathrm{R}$ & $2(14.3)$ & $3(42.9)$ & $1(50)$ & & & & & \\
\hline \multicolumn{9}{|l|}{ CRX } \\
\hline S & 13(92.9) & $6(85.7)$ & $1(50)$ & $2(100)$ & $1(100)$ & & & \\
\hline $\mathrm{R}$ & $1(7.1)$ & $1(14.3)$ & $1(50)$ & & & & & \\
\hline \multicolumn{9}{|l|}{ CPR } \\
\hline S & $12(85.7)$ & $6(85.7)$ & $1(50)$ & $2(100)$ & $1(100)$ & $8(100)$ & $7(87.5)$ & $7(100)$ \\
\hline R & $2(14.3)$ & $1(14.3)$ & $1(50)$ & & & & $1(12.5)$ & \\
\hline \multicolumn{9}{|l|}{ GEN } \\
\hline S & 13(92.9) & $7(100)$ & $1(50)$ & $2(100)$ & $1(100)$ & & $8(100)$ & $6(85.7)$ \\
\hline R & $1(7.1)$ & & $1(50)$ & & & & & $1(14.3)$ \\
\hline \multicolumn{9}{|l|}{ TOB } \\
\hline S & 10(71.4) & $7(100)$ & $1(50)$ & $2(100)$ & $1(100)$ & & & \\
\hline R & $4(28.6)$ & & $1(50)$ & & & & & \\
\hline \multicolumn{9}{|l|}{ TET } \\
\hline S & 7(50) & $4(57.1)$ & & $2(100)$ & & $3(37.5)$ & $3(37.5)$ & $5(71.4)$ \\
\hline $\mathrm{R}$ & $7(50)$ & $3(42.9)$ & $2(100)$ & & $1(100)$ & $5(62.5)$ & $5(62.5)$ & $2(28.3)$ \\
\hline \multicolumn{9}{|l|}{ SXT } \\
\hline S & $6(42.9)$ & $4(57.1)$ & & $2(100 \%)$ & $1(100)$ & & $6(75)$ & $5(71.4)$ \\
\hline $\mathrm{R}$ & $8(57.1)$ & $3(42.9)$ & $2(100)$ & & & & $2(25)$ & $2(28.6)$ \\
\hline \multicolumn{9}{|l|}{ NIT } \\
\hline S & $14(100)$ & $6(85.7)$ & $2(100)$ & 2(100\%) & $1(100)$ & $8(100)$ & $6(75)$ & $7(100)$ \\
\hline $\mathrm{R}$ & & $1(14.3)$ & & & & & $2(25)$ & \\
\hline \multicolumn{9}{|l|}{ NOR } \\
\hline S & $11(78.6)$ & $6(85.7)$ & $1(50)$ & $2(100 \%)$ & $1(100)$ & $5(62.5)$ & $7(87.5)$ & $6(85.7)$ \\
\hline $\mathrm{R}$ & $3(21.4)$ & $1(14.3)$ & $1(50)$ & & & $3(37.5)$ & $1(12.5)$ & $1(14.3)$ \\
\hline \multicolumn{9}{|l|}{ PEN } \\
\hline S & & & & & & $3(37.5)$ & $2(25)$ & $4(57.1)$ \\
\hline $\mathrm{R}$ & & & & & & $5(62.5)$ & $6(75)$ & $3(42.9)$ \\
\hline
\end{tabular}


Table 3 (continued)

\begin{tabular}{|c|c|c|c|c|c|c|c|c|}
\hline \multirow{2}{*}{$\begin{array}{l}\text { Antibiotic } \\
\text { used }\end{array}$} & \multicolumn{8}{|c|}{ Species of isolate with their antibiotic susceptibility } \\
\hline & E. coli $\mathrm{N}(\%)$ & $\begin{array}{l}K . \\
\text { pneumoniae } \\
\mathrm{N}(\%)\end{array}$ & $\begin{array}{l}\text { C. diversus N } \\
(\%)\end{array}$ & E. cloacae N (\%) & $\begin{array}{l}\text { P. mirabilis N } \\
(\%)\end{array}$ & $\begin{array}{l}\text { Enterococci N } \\
\text { (\%) }\end{array}$ & $\begin{array}{l}\text { S. } \\
\text { saprophyticus } \\
\mathrm{N}(\%)\end{array}$ & S. aureus N (\%) \\
\hline \multicolumn{9}{|l|}{ RIF } \\
\hline S & & & & & & $6(75)$ & & \\
\hline $\mathrm{R}$ & & & & & & $2(25)$ & & \\
\hline \multicolumn{9}{|l|}{ VAN } \\
\hline S & & & & & & $7(87.5)$ & & \\
\hline R & & & & & & $1(12.5)$ & & \\
\hline
\end{tabular}

AMP Ampicillin, AMC Amoxicillin-clavulanate, FOX Cefoxitin, CFM Cefixime, CRX Cefuroxime, CPR Ciprofloxacin, GEN Gentamycin, CAZ Ceftazidime, CTX Cefotaxime, RIF Rifampin, NOR Norfloxacin, NIT Nitrofurantoin, PEN Penicillin, RIF Rifampin, SXT Trimethoprim-Sulfamethoxazole, TET Tetracycline, TOB Tobramycin, VAN Vancomycin

Table 4 The prevalence of MDR isolates from urinary stone patients at the University of Gondar Comprehensive Specialized Hospital, January to April 2019

\begin{tabular}{|c|c|c|c|c|c|c|}
\hline \multirow[t]{2}{*}{ Antibiotics } & \multicolumn{5}{|c|}{ Species of bacteria } & \multirow[t]{2}{*}{ Total } \\
\hline & E. coli & K.pneumoniae & C. diversus & Enterococci & S. saprophyticus & \\
\hline AMP, TET, NOR & & & & 2 & & 2 \\
\hline AMP, TET, SXT & & 1 & & & & 1 \\
\hline AMP, AMC, TOB, SXT & 1 & & & & & 1 \\
\hline AMP, TET, TOB, SXT & 1 & & & & & 1 \\
\hline AMP, AMC, TET, SXT & & & 1 & & & 1 \\
\hline AMP, AMC, CTX, SXT & 1 & & & & & 1 \\
\hline CXT, TET, SXT, PEN & & & & & 1 & 1 \\
\hline CXT, TET, NOR, PEN & & & & & 1 & 1 \\
\hline AMP, AMC, CTX, SXT, TET & 1 & & & & & 1 \\
\hline AMP, AMC, CTX, CFM, SXT & 1 & & & & & 1 \\
\hline AMP, FOX, CFM, CPR, NIT & & 1 & & & & 1 \\
\hline AMP, AMC, CRX, TET, SXT & & 1 & & & & 1 \\
\hline AMP, AMC, SXT, GEN, NOR, TET, TOB & 1 & & & & & 1 \\
\hline AMP, AMC, CTX, CFM, GEN, TOB, TET, CPR, SXT, NOR & & & 1 & & & 1 \\
\hline AMP, AMC, CTX, FOX, CFM, CRX, CPR, TOB, TET, NOR & 1 & & & & & 1 \\
\hline Total & 7 & 3 & 2 & 2 & 2 & 16 \\
\hline ESBL positive & 6 & 3 & & & & 9 \\
\hline
\end{tabular}

AMP Ampicillin, AMC Amoxicillin-clavulanate, CTX cefotaxime, FOX Cefoxitin, TET Tetracycline, CPR Ciprofloxacin, CTX Cefotaxime, PEN Penicillin, TOB Tobramycin, SXT Trimethoprim-Sulfamethoxazole, CRX Cefuroxime, GEN Gentamycin, NOR Norfloxacin

[24] and lower than a study done in china $22.0 \%$ [25], Iran 35.5\% [26], Romania 25.8\% [27] and India 45\% [11] among urinary stone patients. However, this result was higher than $7.8 \%$ prevalence of UTI reported from Los Angeles, United States [28]. The variation might be because of the socioeconomic, geographical and population difference. The predominant species isolated in this study were $E$. coli, 14/49(28.6\%) followed by $S$. saprophyticus and Enterococcus species 8/49 (16.3\%) each. The result was comparable to other reports $33 \%$ E.coli and $18.5 \%$ Enterococci isolated from patients with urinary stone [29]. On the other hand the result was higher than 6.5\% prevalence of E.coli reported by Gutierrez et al. [24].

The resistance to antibiotics such as ampicillin, amoxicillin-clavulanate and Trimethoprim-sulphamethoxzole in this study was 31/33(94\%), 18/26(69.23\%) and $17(41.5 \%)$ respectively which was slightly lower than a report in India ampicillin $96 \%$ and amoxicillin-clavulanate $87 \%$ [30]. The result of this study was higher than resistance to Ampicillin 70\%, and Amoxicillinclavulanic acid $30 \%$ reported in Romania [27]. The high rate of resistance might be due to easy access and 
Table 5 Factors associated with UTI among urinary stone patients at the UOGCSH, January to April 2019

\begin{tabular}{|c|c|c|c|c|c|c|}
\hline \multirow[t]{2}{*}{ Variable } & \multicolumn{2}{|c|}{ Significant bacteriuria } & \multirow[t]{2}{*}{ COR 95\% Cl } & \multirow[t]{2}{*}{$P$ value } & \multirow[t]{2}{*}{ AOR $95 \% \mathrm{Cl}$} & \multirow[t]{2}{*}{$P$ value } \\
\hline & Yes (\%) & No (\%) & & & & \\
\hline \multicolumn{7}{|l|}{ Sex } \\
\hline Male & $13(26.5)$ & $140(55.8)$ & 1 & & & \\
\hline Female & $36(73.5)$ & $111(44.2)$ & $3.5(1.77-6.90)$ & 0.00 & $5.76(2.23-13.32)$ & 0.00 \\
\hline \multicolumn{7}{|l|}{ Age } \\
\hline$<20$ years & $3(6)$ & 20(8) & 1 & & 1 & \\
\hline $20-29$ & $21(42.9)$ & 102(40.6) & $1.37(0.37-5.04)$ & 0.63 & $2.00(0.43-9.26)$ & 0.38 \\
\hline $30-39$ & $12(24.5)$ & $85(33.9)$ & $0.94(0.24-3.65)$ & 0.93 & $1.05(0.25-6.57)$ & 0.77 \\
\hline $40-49$ & $9(18.4)$ & $23(9.16)$ & $2.6(0.62-10.98)$ & 0.19 & $2.04(0.38-14.92)$ & 0.36 \\
\hline$\geq 50$ years & $4(8.2)$ & $21(8.4)$ & $1.27(0.25-6.40)$ & 0.77 & $1.25(0.16-12.16)$ & 0.77 \\
\hline \multicolumn{7}{|l|}{ Residence } \\
\hline Urban & $42(85.7)$ & 219(87.3) & 1 & & 1 & \\
\hline Rural & $7(14.3)$ & $32(12.7)$ & $1.14(0.47-2.76)$ & 0.77 & 1 & \\
\hline \multicolumn{7}{|l|}{ Education } \\
\hline Cannot read and write & $10(20.4)$ & $25(10)$ & $2.15(0.90-5.13)$ & 0.08 & $1.80(0.54-4.5 .95)$ & 0.34 \\
\hline Elementary & 13(28.3) & $58(23.1)$ & $1.21(0.56-2.58)$ & 0.63 & $1.42(0.50-4.07)$ & 0.51 \\
\hline High school & $5(10.2)$ & $55(21.9)$ & $0.49(0.18-1.37)$ & 0.17 & $0.31(0.09-1.02)$ & 0.05 \\
\hline College and above & $21(42.9)$ & $113(45)$ & 1 & & 1 & \\
\hline \multicolumn{7}{|l|}{ Family monthly income (ETB) } \\
\hline$\leq 1000$ & $16(32.7)$ & $38(15.1)$ & $2.95(1.413-6.15)$ & 0.004 & $2.40(0.84-6.88)$ & 0.104 \\
\hline $1001-2000$ & $11(22.4)$ & $59(23.5)$ & $1.31(.596-2.857)$ & 0.50 & $1.60(0.61-4.20)$ & 0.34 \\
\hline$>2000$ & $22(44.9)$ & 154(61.4) & 1 & & 1 & \\
\hline \multicolumn{7}{|l|}{ History of UTI } \\
\hline Yes & 15(30.6) & $18(7.2)$ & $5.71(2.63-12.38)$ & 0.00 & $4.66(1.76-12.30)$ & 0.002 \\
\hline No & $34(69.4)$ & 233(92.8) & 1 & & 1 & \\
\hline \multicolumn{7}{|l|}{ Urinary blockage } \\
\hline Yes & $3(6.1)$ & $6(2.4)$ & $2.66(0.64-11.03)$ & 0.18 & $0.26(0.03-2.20)$ & 0.22 \\
\hline No & 46(93.9) & 245(97.6) & 1 & & 1 & \\
\hline \multicolumn{7}{|l|}{ Stone size } \\
\hline$<5 \mathrm{~mm}$ & 43(87.8) & 232(92.4) & 1 & & & \\
\hline$\geq 5$ & $6(12.2)$ & $19(7.6)$ & $1.7(0.64-4.5)$ & 0.28 & & \\
\hline \multicolumn{7}{|l|}{ Stone location } \\
\hline Renal & 46(93.9) & $236(94)$ & 1 & & & \\
\hline Renal and/or extra renal & $3(6)$ & $15(6)$ & $1.03(0.29-3.7)$ & 0.97 & & \\
\hline \multicolumn{7}{|l|}{ Chronic disease } \\
\hline Yes & $6(12.2)$ & $15(6)$ & $2.2(0.81-1.5 .97)$ & 0.12 & $1.17(0.24-5.65)$ & 0.85 \\
\hline No & 43(87.8) & $236(94)$ & 1 & & 1 & \\
\hline \multicolumn{7}{|l|}{ Drug use } \\
\hline Yes & $36(73.5)$ & 136(54.2) & 1 & & 1 & \\
\hline No & $13(26.5)$ & 115(45.8) & $0.43(0.22-0.84)$ & 0.014 & $0.33(0.14-0.77)$ & 0.01 \\
\hline \multicolumn{7}{|l|}{ Hospitalization } \\
\hline Yes & $7(14.3)$ & $10(4)$ & $4.02(1.45-11.14)$ & 0.008 & $3.85(0.92-16.07)$ & 0.065 \\
\hline No & $42(85.7)$ & $241(96)$ & 1 & & 1 & \\
\hline \multicolumn{7}{|l|}{ Sexual activity } \\
\hline Within 2 days & $6(12.2)$ & $37(14.7)$ & $0.55(0.21-1.45)$ & 0.23 & $0.61(0.20-1.92)$ & 0.40 \\
\hline Within 7 days & $11(22.4)$ & $73(29.1)$ & $0.51(0.24-1.10)$ & 0.09 & $0.48(0.19-1.21)$ & 0.12 \\
\hline Within a month & $5(10.2)$ & $49(19.5)$ & $0.35(0.13-0.96)$ & 0.041 & $0.30(0.086-1.02)$ & 0.054 \\
\hline Never & $27(55.1)$ & $92(36.7)$ & 1 & & 1 & \\
\hline BMl & & & & & & \\
\hline $18.5-24.5$ & $32(65.3)$ & 179(71.3) & 1 & & 1 & \\
\hline$>24.5$ & $7(14.3)$ & $42(16.7)$ & $0.536(0.24-1.20)$ & 0.13 & $1.24(0.41-3.79)$ & 0.71 \\
\hline$<18.5$ & 10(20.4) & $30(12)$ & $0.5(0.17-1.46)$ & 0.21 & $2.89(0.98-8.50)$ & 0.053 \\
\hline
\end{tabular}


self-medication, weak adherence of patients to prescribed antimicrobial agents, wider availability of empirical treatment in health care settings [12, 31, 32]. Most of the isolates identified were susceptible to nitrofurantoin 46/49(94\%) and ciprofloxacin 44/49(90\%). There were two methicillin resistant S. saprophyticus and one methicillin resistant $S$. aureus (MRSA) which was nearly similar to a case of methicillin resistant coagulase negative staphylococcus report in Romania [27].

From a total of 16 MDR isolates, E. coli 7/16(43.75\%) and $K$. pneumoniae $3 / 16(18.75 \%)$ were the most common MDR species. The MDR isolates in this study were higher than $29.3 \%$ MDR E. coli, $6.8 \%$ K. pneumoniae but lower compared to $12.0 \%$ MDR E. faecalis. The difference could be due to sample size variation [33]. Furthermore, 9/26(34.6\%) of the total 26 Enterobacteriaceae, were ESBL producing species. These ESBL producing species were $E$. coli 6/26(23\%) and $K$. pneumoniae $3 / 26(11.5 \%)$ species. This result was lower than $51.8 \%$ of the overall prevalence of ESBL producing Enterobacteriaceae [12]. The prevalence of ESBL producing Enterobacteriaceae from the total E.coli isolates was higher than $8.7 \%$ result by Bianca et al. [31]. This might be due to variation in the management and use of antibiotics.

In the present study the prevalence of UTI was higher among female participants $36 / 49$ (73.5\%). This corresponds to a study done by Bianca et al. [31] 74.2\% in females and $25.8 \%$ in males. The result was slightly higher than a $67 \%$ prevalence in females as compared to $33 \%$ in males [11]. The likelihood of acquiring UTI in females was 5.76 times higher compared to males (AOR 5.76, 95\% CI 2.23-13.32). This could be due to the physio-anatomical variations between males and females including anal and genital proximity, short urethra as well as sexual activity in females which ease the access of intestinal flora to the urinary tract in females. On the other hand antimicrobial activity of the prostate secretions in males could contribute to the reduced infection rate among males than female participants [25, 27].

The finding of this study showed that $15 / 49$ (31\%) of patients with significant bacteriuria had previous history of UTI. Having history of UTI increases the risk of UTI by 4.7 times (AOR 4.66, 95\% CI 1.76-12.30) than those who had not. This might be due to the release of bacteria from the inside of stone or from its matrix protected surface resulting in persistence or recurrence of the UTI. Previous history of UTI is among the factors that predisposes to the occurrence of the infection [15]. The treatment might not have completely removed the etiologic agent of UTI in patients. Because the bacteria can be incrusted in the stone or extracellular matrix and escape from the treatment [34].

In addition, 36/49(73.5\%) patients with significant bacteriuria had previous history of drug use and showed a statistically significant association with UTI. The risk of UTI among patients who had not history of drug use reduces by $67 \%$ compared to those who had history of drug use (AOR 0.33, 95\% CI 0.14-0.77). This could be due to the fact that steroidal drugs are immunosuppressive and patients could be immunocompromised [30]. The increased antimicrobial resistance in drug users and the recurrence or persistence of drug resistant UTI might also contribute.

As a limitation only urine specimen was taken for culture and stone culture was not performed to which could help to know the agreement of stone and urine culture strain characterization was not performed due to lack of resources.

\section{Conclusion}

The commonest bacterial species isolated from patients with urinary stone disease was $E$. coli. The most common MDR and ESBL producing isolates were E. coli and $K$. pneumoniae. Being female, previous history of UTI and drug use were the independent risk factors. Isolated uropathogens were most susceptible to nitrofurantoin, ciprofloxacin and gentamycin. However, most of the isolates were resistant to ampicillin, penicillin and trimethoprim-Sulfamethoxazole. Routine diagnosis of urinary stone patients for urinary tract infection should be promoted. Antimicrobial stewardship program should be implemented to reduce drug resistance.

\section{Supplementary Information}

The online version contains supplementary material available at https://doi. org/10.1186/s12894-021-00794-8.

Additional file 1. English version of the questionnaire.

\section{Abbreviations}

AST: Antimicrobial susceptibility test; ATCC: American Type Culture Collection; BAP: Blood agar plate; CLSI: Clinical Laboratory Standards Institute; CLED: Cysteine lactose electrolyte deficient agar; ESBL: Extended spectrum beta lactamase; HAART: Highly active antiretroviral therapy; MAC: MacConkey 
agar; MDR: Multidrug resistance; TSI: Triple sugar iron agar; USD: Urinary stone disease; UTI: Urinary tract infection.

\section{Acknowledgements}

We would like to acknowledge study participants for their cooperation and the University of Gondar for financial and material support.

\section{Authors' contributions}

DK: Conception of the research idea, study design, data collection, data analysis, interpretation, write up of the research. ZT, FM and SE: supervision and review of the manuscript: AD: Data collection and review of the manuscript. All authors read and approved the final manuscript.

\section{Funding}

University of Gondar has funded this research work.

\section{Avaliability of data and materials}

The datasets used and/or analyzed during the current study are available from the corresponding author on reasonable request.

\section{Ethical approval and consent to participate}

Ethical approval was obtained from an Ethical review committee of the School of Biomedical and Laboratory Sciences, college of Medicine and health sciences, University of Gondar (SBMLS/2923/11). The study was conducted in accordance with the ethical principles of the declaration of Helsinki on human subjects. The purpose of the study, the possible benefits and risks on participants were explained to the study prticipants and participation was fully voluntary. Written informed consent was obtained from the study participants and assent was sought from families or legal guardians of under 18 years children. All the ethical standards were followed. Positive cases were connected to physicians for management.

\section{Consent for publication}

Not applicable.

\section{Competing interests}

Authors declare that there is no competing interest.

\section{Author details}

1 Department of Medical Microbiology, School of Biomedical and Laboratory Sciences, College of Medicine and Health Sciences, University of Gondar, P.O. Box 196, Gondar, Ethiopia. ${ }^{2}$ Department of Radiology, School of Medicine, College of Medicine and Health Sciences, University of Gondar, P.O. Box 196, Gondar, Ethiopia.

\section{Received: 27 November 2020 Accepted: 1 February 2021}

Published online: 23 February 2021

\section{References}

1. Schwaderer AL, Wolfe AJ. The association between bacteria and urinary stones. Ann Transl Med. 2017:5(2):32

2. Kirkali Z, Rasooly R, Star RA, Rodgers GP. Urinary stone disease: progress, status, and needs. Urology. 2015;86(4):651-3.

3. Sakhaee K, Maalouf NM, Sinnott B. Kidney stones 2012: pathogenesis, diagnosis, and management. J Clin Endocrinol Metab. 2012;97(6):1847-60.

4. Miano R, Germani S, Vespasiani G. Stones and urinary tract infections. Urologia Int. 2007;79(Suppl. 1):32-6.

5. Thomas B, Tolley D. Concurrent urinary tract infection and stone disease: pathogenesis, diagnosis and management. Nat Rev Urol. 2008:5(12):668-75.

6. Hassan JK. Crystalluria types and incidence in Basra City; southern of Iraq. J Basrah Res (Sciences). 2011;37(5A):72-81.

7. Shojaeian A, Rostamian M, Noroozi J, Pakzad P. The identification of chemical and bacterial composition and determination of FimH gene frequency of kidney stones of Iranian patients. J Res Med Sci. 2016;18(6):e7363.
8. Flannigan R, Choy WH, Chew B, Lange D. Renal struvite stones-pathogenesis, microbiology, and management strategies. Nat rev Urol. 2014;11(6):333-41.

9. Norsworthy AN, Pearson MM. From catheter to kidney stone: the uropathogenic lifestyle of P. mirabilis. Trends Microbiol. 2017;25(4):304-15.

10. Romanova YM, Mulabaev N, Tolordava E, Seregin A, Seregin I, Alexeeva N, et al. Microbial communities on kidney stones. Mol Gen Microbiol Virol. 2015;30(2):78-84

11. Songra M, Damor M, Namdev RK, Patbamniya NK, Nawalakhe P, Jain R. A study on positive stone culture and its association with rate of sepsis after urological procedures. Int Surg J. 2016;2(2):239-46.

12. Chen D, Zhang Y, Huang J, Liang X, Zeng T, Lan C, et al. The analysis of microbial spectrum and antibiotic resistance of uropathogens isolated from patients with urinary stones. Int J Clin Pract. 2018;72(6):e13205.

13. Raheem OA, Khandwala YS, Sur RL, Ghani KR, Denstedt JD. Burden of urolithiasis: trends in prevalence, treatments, and costs. Eur Urol Focus. 2017;3(1):18-26.

14. Scales CD Jr, Smith AC, Hanley JM, Saigal CS. Prevalence of kidney stones in the United States. Eur Urol. 2012;62(1):160-5.

15. Foxman B. Urinary tract infection syndromes: occurrence, recurrence, bacteriology, risk factors, and disease burden. Infect Dis Clin North Am. 2014;28(1):1-13.

16. Tandogdu Z, Wagenlehner FM. Global epidemiology of urinary tract infections. Curr Opin Infect Dis. 2016;29(1):73-9.

17. Eshetie S, Unakal C, Gelaw A, Ayelign B, Endris M, Moges F. Multidrug resistant and carbapenemase producing Enterobacteriaceae among patients with urinary tract infection at referral Hospital, Northwest Ethiopia. Antimicrob Resist Infect Control. 2015;4(1):12.

18. Cheesbrough M. District laboratory practice in tropical countries. Cambridge: Cambridge University Press; 2006.

19. CLSI. Performance Standards for Antimicrobial Susceptibility Testing. 28th ed. CLSI supplement M100. 2018.

20. Tellevik MG, Blomberg B, Kommedal Ø, Maselle SY, Langeland N, Moyo SJ. High prevalence of faecal carriage of ESBL-producing Enterobacteriaceae among children in Dar es Salaam, Tanzania. PLoS ONE. 2016:11(12):e0168024.

21. Goyal D, Dean N, Neill S, Jones P, Dascomb K, editors. Risk Factors for Community-Acquired Extended-Spectrum Beta-Lactamase-Producing Enterobacteriaceae Infections-A Retrospective Study of Symptomatic Urinary Tract Infections. In: Open forum Infect Dis; 2019; 6(12):ofz517. Oxford University Press.

22. Pasricha J, Koessler T, Harbarth S, Schrenzel J, Camus V, Cohen G, et al. Carriage of extended-spectrum beta-lactamase-producing enterobacteriacae among internal medicine patients in Switzerland. Antimicrob Resist Infect Control. 2013;2(1):20.

23. Kin-On K, Emily C, Chung P-H, Arthur T, Wan-In W, Chendi Z, et al. Prevalence and associated factors for carriage of Enterobacteriaceae producing ESBLs or carbapenemase and Methicillin-resistant Staphylococcus aureus in Hong Kong Community. J Infect. 2020;81(2):242-7.

24. Gutierrez J, Smith A, Geavlete P, Shah H, Kural AR, de Sio M, et al. Urinary tract infections and post-operative fever in percutaneous nephrolithotomy. World J Urol. 2013;31(5):1135-40.

25. Yongzhi L, Shi Y, Jia L, Yili L, Xingwang Z, Xue GJ. Risk factors for urinary tract infection in patients with urolithiasis primary report of a single center cohort. BMC Urol. 2018;18(1):45.

26. Shafi H, Shahandeh Z, Heidari B, Sedigiani F, Ramaji AA, Pasha YRY, et al. Bacteriological study and structural composition of staghorn stones removed by the anatrophic nephrolithotomic procedure. Saudi J Kidney Dis Transpl. 2013;24(2):418-23.

27. Maier A, Man A, Chibelean C, Cighir T, Nemes-Nagy E, Maier I, et al. Bacteriological evaluation of the non-struvite nephrolithiasis and its association with urinary tract infections. Rev Rom Med Lab. 2015;23(4):457-68.

28. Abrahamian FM, Krishnadasan A, Mower WR, Moran GJ, Talan DA. Association of pyuria and clinical characteristics with the presence of urinary tract infection among patients with acute nephrolithiasis. Ann Emerg Med. 2013;62(5):526-33.

29. Tavichakorntrakool R, Prasongwattana $V$, Sungkeeree S, Saisud P, Sribenjalux P, Pimratana C, et al. Extensive characterizations of bacteria isolated from catheterized urine and stone matrices in patients with nephrolithiasis. Nephrol Dial Transplant. 2012;27(11):4125-30. 
30. Bora DI, Lyngdoh DW, Khyriem DB. Bacteriological profile of rena. Int J Curr Res. 2015;7(6):16898-901.

31. Bianca T, Adrian M, Emil M, Adrian T. Microbiological study of urinary calculi in patients with urinary infections. Acta Medica Transilv. 2013:2(2):245-9.

32. Bell BG, Schellevis F, Stobberingh E, Goossens H, Pringle M. A systematic review and meta-analysis of the effects of antibiotic consumption on antibiotic resistance. BMC Infect Dis. 2014;14(1):13.

33. Wang $S$, Zhang $Y$, Zhang $X$, Li J. An evaluation of multidrug-resistant (MDR) bacteria in patients with urinary stone disease: data from a highvolume stone management center. World J Urol. 2020;38(2):425-32.
34. Hedelin $\mathrm{H}$. Uropathogens and urinary tract concretion formation and catheter encrustations. Int J Antimicrob Agents. 2002;19(6):484-7.

\section{Publisher's Note}

Springer Nature remains neutral with regard to jurisdictional claims in published maps and institutional affiliations.
Ready to submit your research? Choose BMC and benefit from:

- fast, convenient online submission

- thorough peer review by experienced researchers in your field

- rapid publication on acceptance

- support for research data, including large and complex data types

- gold Open Access which fosters wider collaboration and increased citations

- maximum visibility for your research: over $100 \mathrm{M}$ website views per year

At BMC, research is always in progress.

Learn more biomedcentral.com/submissions 